\title{
The Application of Relevance Theory in English News Translation
}

\author{
Rongle Yao \\ English Department, School of Public Fundamentals, Jiangsu Vocational College of Medicine, \\ Yancheng 224006, China
}

Keywords: Relevance theory; English news; Translation; Application

\begin{abstract}
In the context of the development of economic globalization, the exchanges between countries and countries are getting closer and closer, and the ability of English news translation is becoming more and more important. English news translation work is an important language communication method in economic globalization, which provides strong support for communication and exchange between countries. Relevance theory makes English news translation develop to standardization, and finds the communicative relationship between different languages with the necessary relevance principle, so that readers can understand the actual intention of the author and ensure that English news translation can accurately translate the actual content.
\end{abstract}

\section{Introduction}

Today, with the continuous development of the global economy, some domestic industrial chain extension enterprises and foreign companies have reached a consensus on cooperation and joint ventures, and news communication provides a bridge for the exchange of information and rational communication in economic and cultural exchanges. The continued development of the economy. However, there are certain obstacles between different languages and cultures, and the translation of language can achieve reasonable communication between the state and the country. The emergence of relevance theory has laid a solid theoretical foundation for English news translation. Based on the theory of relevance, the article analyzes the translation of English news, and on the basis of ensuring the high-quality relevance of English translation, the readers of the translation can correctly understand the meaning of the news.

\section{The development of English news translation under the influence of relevance theory}

Relevance theory was proposed as early as 1986, and was proposed by Dan Sperber (French) and Dill Daryl Wilson (English) in "Relationship: Communication and Cognition", which summarizes human language communication activities. A conscious cognitive activity for the unknown. People's language communication is also a process of reasoning communication. It is analyzed by the related dialogues and cognitive things between things, and then consensus is reached to deepen understanding. Relevance theory researches and analyzes the understanding of different languages in a scientific cognitive way, and extends it to the understanding of different language conceptual 
and linguistic environments, and then optimizes the mechanism of reasoning.

Relevance is divided into maximum relevance and optimal relevance. For optimal relevance, it is mainly for the communicative content of the language. The English news translation work under the theory of relevance is designed into the verbal reasoning process of the translator's ideological mechanism. Through the efforts of English news translators, the best correlation between different language communication is found. The translator's translation makes the recipient of the translated information. It is easy to understand the language environment of language communication and realize the accurate transmission of information in different languages [1].

\section{The particularity of English news and its translation}

For English journalism, influenced by the particularity of its language, the corresponding news will also show certain uniqueness. The English language has obvious characteristics of purpose, timeliness and factuality, and it often directly reflects the intention of life activities of the source of language. For the language of English news, the differences between different languages and cultures will lead to the unique characteristics of English news as a whole. For example, the short and popular language features of English news show the reader easy to understand and suit. Therefore, when translating English news, it is necessary to distinguish between the translation work of ordinary English articles. The translation work of English news cannot be translated according to the translation skills in traditional translation work, such as "faithful, fluent and beautiful” and so on. It does not apply to the translation of English news. The translation of English news should ensure that the translation can change the meaning of the original English news, and on the basis of loyalty to the news itself, the reader's ability to understand and accept the news content is considered [2].

In the actual English news translation work, we must master the different characteristics of English news translation, and carry out translation work reasonably. For example, in English news translation, full-text translation can be carried out according to different English news secondary transmissions. In the news translation, according to the actual understanding needs, the original English news sentences and words should be appropriately cut or changed in order to make them more It is easy to be understood by a wide audience. At the same time, it is necessary to recognize the reasonable transmission of English news translation knowledge to English news. It is not possible to deliberately change the meaning expressed by the news, and to ensure that the translated questions accurately represent the news content. In addition, English news translation requires translators to have the ability to compile and compile appropriate news content based on the understanding of English news to help readers understand English news content more smoothly. For example, use some modern words to enrich English news content and give readers a good reading experience. UNESCO (United Nations Educational, Scientific and Cultural Organization) UNESCO can use modern acronyms to replace it, reduce the burden of reading for readers, and pass on the actual "UNESCO" information to readers.

\section{English news translation practice supported by relevance theory}

Translation of English news is a language communication between translators and readers. However, it requires readers to have a certain understanding of the meaning of English news translation, and can imagine and reason the news content according to the discourse of English news translation. For English news translators, they can standardize the translation of English news with the support of relevance theory, give the readers a fully understood target language translation text, and fully convey the English news information content to the readers, readers. Think about the corresponding information language environment, and then have a deep understanding of English 
news, so that the correlation effect is maximized.

\subsection{English news style composition}

English news consists of three parts: title, quotation and subject. For the title of English news, it is mainly the content of the news as a whole, and the topic of the news is the overall meaning of the news. The topic of news can reflect the essence of news content, and it is characterized by simplicity and conspicuousness. For news quotes, it is generally the first part of the news, refining the overall content of the news, evoking the reader's interest in reading the news content with vivid statements, and letting the reader have a systematic view of the actual news content. The outline of the understanding. The main body of English news is to describe the accuracy of actual news events, and to describe the production and details of news. For the English language, the main news content describes the overall content of the event in an easy-to-understand language.

\subsection{Title Translation}

In the translation of the title, the translation work is required to choose the appropriate translation method between translation descriptiveness and interpretability. For English news, its own title translation and content translation have certain differences. The slogan title itself has certain relevance to the news topic, and at the same time it must have certain distinctions, so as to avoid the original communicator and the reader of the translation. There is a differential understanding of the meaning of the news. Make sure that English news headlines reflect true and reliable news information. For example, for The Madrid government also hope sitsnewcoopera? Tionagreement with Moroccomay help strengthen the flow. In the translation of The New York Times/Oct, 10, 2004), "Madrid" means Spain, and the overall sentence is translated as "The Spanish government hopes that the new cooperation agreement with Morocco can effectively curb the influx of illegal immigrants". "the flow" means the flowofillegalmigrants. In the translation of the title of English news, different forms of translation may cause readers to misunderstand the actual English news content, and doubt the title of English news after understanding the main content of English news. Therefore, the translation of English news should be carried out under the support of relevance theory, combined with the readers and English news communicators, and it is possible to ensure a smooth connection as much as possible on the basis of the original translation of English news. Or delete appropriate words to enable the readers to better understand the true content of English news.

\subsection{Text translation}

The translation of the English news text should focus on the connection between the title and the text. The translator should reasonably reflect the meaning of the surface language expression and the meaning of the implicit event in English news based on the actual meaning of English news. Understand, the descriptive meaning of the original author and the concealed meaning are reasonably expressed [3]. This requires the translators of English news to refine various information in English news, summarize the characteristics after reasonable analysis of the information, make reasonable adjustments to different information, and make obvious meanings and hidden meanings to the news authors by combining old and new information. The meaning of sexuality is expressed, so that the translator can express the true meaning of the author's original meaning in the original work based on the understanding of the language, and then realize the cognition of the English journalism work, the original author, and the majority of the reading group. The best correlation between sexual language environments. 


\section{Discussion on English News Translation Skills Supported by Relevance Theory}

In the actual translation and work of English news, translators must rely on the relevance theory to reduce the differences and obstacles between English and native language with the support of relevance theory, by finding the optimal relationship between the two. Sexuality, reducing the impact of cultural differences on language translation and language understanding. Through the application of relevance theory, on the basis of ensuring the authenticity and reliability of translated English news, the readers can realize the relevance, find the best relevance, and achieve the best communicative effect in English. In order to achieve the ideal English news translation effect, the corresponding English news translators need to use professional technical skills in the actual translation work to conduct professional analysis of English news content.

\subsection{Add translations as appropriate}

Among the additions to translation, most of them are in the original English news content. The cohesiveness or linguistic narrative between some sentences and the language and culture of the translated language are obviously different. On the basis of recognizing the differences, in order to facilitate the readers to be able to understand English news content more, you need to add translations appropriately. In addition, different readers in the readership have different knowledge backgrounds and different levels of understanding of English news. Therefore, it is necessary to add translations and explanations of details to the translation of English news to help more readers understand the actual content of English news. For example, in the actual English news translation work, the content of English history, culture, social background, language features, etc., readers may have questions about the translation content of this category, and cannot correctly understand English news content, which requires corresponding English. The news translators explain the different regional cultures in detail, and explain the contents of some English cultures that need to be explained in the form of annotations below the English news, so that the actual expression of the original news creators and the readers of the target audience Looking forward to maintaining consistency, we can deepen readers' cognition of content, analyze the problems in English news, analyze the rationality and particularity of the problem under the British cultural background and folk customs, and then create the unity with the original news creators. .

\subsection{Recombinant translation}

Recombinant translation is a more practical work in English news translation. Through the reorganization of English language and sentences, the English language expression is translated into English language translation and translated into language expressions acceptable to the masses of readers. Then it is obvious to understand the actual content of English news. When translating English news, the use of language culture and target language in the original English news is carried out around the communication and daily life habits of English itself, but for the translation work of English news, the corresponding audience is not In the daily life of English-language translation work, translators are required to analyze the similarities and differences between Chinese and English in English news based on their own understanding, and connect them in different places with bold compiling methods. And replace, thereby reducing the difference between the English language and Chinese culture, expression techniques, and find the best correlation between the two. In the actual work, there is a big difference between the English sentence and the Chinese sentence. The structure of the two, the length of the sentence, and the order of use of the corresponding words have certain differences, which in turn leads to some special translation work. The sentence is optimized and improved, and the word arrangement of the original text is adjusted to translate the 
English news content suitable for the mass audience. For example, "One of the Lost Tribes of Israel Surfaces in India" means that a forgotten Israeli tribe appears in India, "appears" in the Chinese expression at the end, but in English "India" is placed at the end of the sentence. According to the logic of Chinese and the vocabulary usage habits, the actual content of English news can be adjusted to make the content that the original author of English news want to express can be unified with the understanding content of the majority of English news translation audience.

\subsection{Innovative translation}

Innovative translation technology is an efficient English news translation technique. For Chinese and English, the language form and language culture vary widely. For example, Chinese and English have different expressions of the subject. The differences between their respective ways of thinking, social customs and values determine the actual English news. It is not possible to translate in accordance with the English habits and language expression order. On the basis of understanding, follow the principle of "letter, dad, and elegance" to carry out innovative translation of the original English news content.

\section{Conclusion}

In summary, the relevance theory holds that human language communication belongs to a kind of reasoning dynamic communication, and the actual translation work of English news is also a kind of language communication. The application analysis of relevance theory in English news translation, first of all Analyze the content of relevance theory and the particularity of English news and its translation content. After understanding the content of relevance theory and the particularity of English news and its translation content, study the English news translation practice supported by relevance theory, and analyze the specific association. The theory of English news translation is supported by the theory of sex. Through the analysis and discussion of the whole process, English news translators can be effectively supported to carry out their own work.

\section{References}

[1] Li Xia. Practice and Thinking of Relevance Theory in English News Translation Journal of Jiamusi Vocational College, Vol.1 (2016) No.15, p. 380-381.

[2] Huo Yanjing. Analysis of News Translation Methods under the Guidance of Relevance Theory. Journal of Liaoning Provincial College of Communications, Vol.6 (2017) No.22, p. 34-37.

[3] He Lina. A Brief Discussion on the Countermeasures of English Translation of Nanchang Red Tourism Text under Relevance Theory. Science \& Technology Information, Vol.14 (20164) No.34, p. 212-213. 